\title{
CARACTERIZAÇÃO DAS ÓRTESES UTILIZADAS POR CRIANÇAS COM PARALISIA CEREBRAL ATENDIDAS NO CENTRO DE REABILITAÇÃO FÍSICA EM CASCAVEL-PR
}

\author{
CHARACTERIZATION OF THE ORTHOSIS USED BY CHILDREN WITH CEREBRAL PALSY ASSISTED \\ IN THE PHYSICAL REHABILITATION CENTRE IN CASCAVEL-PR
}

\author{
Danilo de Oliveira Silva $a^{a^{*}}$, Marcella Ferraz Pazzinatto ${ }^{b^{* *}}$, Aniele Tomadon ${ }^{\mathrm{c}^{* *}}$, \\ Álvaro José Mayer Ferreira ${ }^{\mathrm{d}^{* *}}$, Anamaria Meireles ${ }^{\mathrm{e}^{* * *}}$, Jane Aparecida de Oliveira Silva ${ }^{\mathrm{f} \#}$ \\ adanilo110190@hotmail.com, bferraz_mar@hotmail.com, canitomadon@hotmail.com, \\ dalvamayer@hotmail.com, eanammeireles@yahoo.com.br, fjaneolives@hotmail.com \\ "Universidade Estadual Paulista "Júlio de Mesquita Filho" - Presidente Prudente \\ "Universidade Estadual do Oeste do Paraná \\ ${ }^{* * *}$ Universidade Federal de Santa Catarina \\ "Universidade do Vale do Sapucaí.
}

Data de entrega do artigo: 17/11/2013

Data de aceite do artigo: 04/06/2014

\section{RESUMO}

Introduçáo: A paralisia cerebral é definida como um distúrbio permanente e variável do movimento e da postura, devido a uma lesão não progressiva do cérebro no período pré, peri ou pós-natal. $\mathrm{O}$ uso de órteses destaca-se como um recurso importante no processo de reabilitaçáo e tem por objetivos proteger a cicatrização de estruturas, manter ou promover a amplitude de movimento de determinada articulação, substituir ou aumentar uma função, prevenir ou corrigir deformidades, oferecer repouso articular e reduzir a dor. Objetivo: Realizar um levantamento sobre os tipos e as características da utilizaçáo das órteses pelas crianças com paralisia cerebral atendidas no setor de pediatria do Centro de Reabilitaçáo Física (CRF) da Universidade Estadual do Oeste do Paraná (Unioeste). Materiais e Métodos: Foi aplicado um questionário pré-formulado a fim de obter informaçôes pessoais, identificação do tipo de órtese utilizada em cada criança, tempo e forma de uso; o questionário foi respondido pelos pais ou responsáveis da criança. Resultados: Todas as 35 crianças avaliadas usam órteses de diferentes tipos; $60 \%$ delas fazem uso das órteses no período noturno, e 10 crianças utilizam meios auxiliares de locomoção. Conclusão: A órtese mais utilizada é a suropodálica sem articulação, e o tempo médio diário de permanência com a órtese é de 13 horas e 54 minutos, sendo que a maior parte das crianças faz uso da órtese no período noturno.

Palavras-chave: Órteses; paralisia cerebral; reabilitação

\section{ABSTRACT}

Introduction: Cerebral palsy is defined as a permanent and variable disorder of movement and posture due to a non-progressive brain injury in pre, peri or post-natal. The use of orthosis stands out as an important resource in the rehabilitation process and aims to protect healing structures, maintain or promote the range of motion of a particular joint, replace or improve a function, prevent or correct deformities, to offer joint rest and reduce pain. Objective: To conduct a survey of the types and characteristics of the use of orthosis for children with cerebral palsy treated at the pediatric sectorof the Physical Rehabilitation Centre (FRC) of the State University of West Paraná (Unioeste). Materials and Methods: A questionnaire pre-formulated with personal information identifying the type of prosthesis used in each child, time and manner of use; the questionnaire was completed by parents or guardians of the child. Results: All the 35 children evaluated use orthosis of different types; $60 \%$ of them use orthosis in the evening, and 10 children use mobility aids. Conclusion: The most commonly used orthoses is the sural-podal without linkage, and the average length of use is 13 hours and 54 minutes per day; most children use the orthoses at night.

Keywords: Orthosis; cerebral palsy; rehabilitation. 


\section{Introdução}

A paralisia cerebral (PC) é definida como um distúrbio permanente e variável do movimento e da postura devido a uma lesáo náo progressiva do cérebro no começo da vida ${ }^{1}$. É consequência de uma lesão ocorrida no período pré, peri ou pós-natal que afeta o sistema nervoso central em fase de maturação estrutural e funcional ${ }^{2}$. Os distúrbios encontrados nas crianças com paralisia cerebral caracterizam-se pela falta de controle sobre os movimentos e por modificaçóes adaptativas do comprimento muscular, resultando, em alguns casos, em deformidades ósseas ${ }^{3}$.

A PC é uma condição que frequentemente interfere no aprendizado de habilidades motoras na infância, as quais são essenciais para o desempenho de quaisquer atividades de vida diária (AVDs). As mudanças de posturas geralmente apresentam-se alteradas em crianças com PC quando comparadas a crianças normais ${ }^{4}$.

Ao considerar as capacidades motoras das crianças com PC, as demandas da tarefa e as características do ambiente, observa-se que, muitas vezes, há necessidade de melhores mudanças nas posturas. Entretanto, a forma como elas as realizam pode colocá-las em situaçáo de risco quanto a futuras alteraçóes estruturais, como o aparecimento de encurtamentos, deformidades e prejuízo futuro na dinâmica da marcha. Com isso, as órteses de posicionamento são utilizadas para auxiliar no tratamento de crianças portadoras de paralisia cerebral. O suporte mecânico oferecido pelas órteses visa minimizar ou corrigir o padrão não fisiológico de marcha assumido por algumas dessas crianças, proporcionando benefícios como a melhora da qualidade de deambulação e a prevenção de deformidades ${ }^{5}$.

$\mathrm{O}$ uso de órteses destaca-se como um recurso importante no processo de reabilitação e tem por objetivos: proteger a cicatrização de estruturas; manter ou promover a amplitude de movimento de determinada articulação; substituir ou aumentar uma função; prevenir ou corrigir deformidades; oferecer repouso articular; reduzir a dor ${ }^{6,7}$. A fabricação das órteses é feita de forma individualizada e considera vários fatores, como a fisiopatologia, a biomecânica articular, a função e as necessidades do paciente ${ }^{8}$.

É imprescindível que a orientação sobre o uso correto da órtese seja exaustivamente discutida com os pais ou responsáveis pela criança, tanto quanto o período (noturno, diurno ou ambos), o tempo de uso diário e o tempo pelo qual a órtese será utilizada até o momento da sua substituição.

Tendo isso em vista, o objetivo do presente estudo é realizar um levantamento sobre os tipos e as características de utilização das órteses pelas crianças com paralisia cerebral atendidas no setor de pediatria do Centro de
Reabilitaçáo Física (CRF) da Universidade Estadual do Oeste do Paraná (Unioeste).

\section{Materiais e métodos}

\section{Amostra}

A amostra foi composta por 35 crianças com idade média de 5,2 $\pm 1,8$ anos, portadoras de paralisia cerebral que fazem o uso de órtese, cadastradas para atendimento no CRF da Unioeste. Os responsáveis pelas crianças foram convidados a participar do estudo e assinaram, em duas vias, o Termo de Consentimento Livre e Esclarecido (TCLE) previamente aprovado pelo Comitê de Ética em Pesquisa da Unioeste (Parecer nº 96.908).

\section{Critérios de Inclusão}

Foram incluídas no estudo todas as crianças atendidas pelo CRF da Unioeste com diagnóstico clínico de paralisia cerebral as quais fazem uso de órteses.

\section{Critérios de Exclusão}

Foram excluídas do estudo as crianças cujos responsáveis não concordaram em assinar o TCLE.

\section{Procedimentos}

Os procedimentos do estudo foram desenvolvidos no Centro de Reabilitação Física da Universidade Estadual do Oeste do Paraná, na cidade de Cascavel (PR). As coletas de dados foram realizadas na sala de espera do CRF, durante o atendimento das crianças.

Trata-se de um estudo descritivo transversal, em que foram analisados os tipos de órteses por meio de consulta aos prontuários de pacientes com diagnóstico de paralisia cerebral do setor de pediatria do CRF/Unioeste atendidos no primeiro semestre de 2013. Os dados relativos ao uso (quantidade de horas diárias, período e tempo de uso) foram obtidos por meio de entrevista com os responsáveis pelo paciente, na qual se utilizou um questionário pré-formulado, o qual continha informaçóes sobre os tipos de órteses utilizados, o tempo e a forma de uso.

\section{Análise Estatística}


Para a análise estatística, os dados foram previamente tabulados no programa Microsoft Office Excel', versão 2007. Em seguida, a análise estatística foi iniciada pela exploraçáo de dados descritivos; após esse momento, foi realizada uma estatística percentual dos dados e exposiçáo em forma de gráficos.

\section{Resultados}

As crianças permanecem com as órteses, em média, por 13 horas e 54 minutos por dia. O período de uso noturno das órteses corresponde a 29 crianças (60\%), e o diurno, a 19 crianças (40\%) (Figura 1).

Figura 1: Porcentagem de crianças que fazem uso de órtese durante o período diurno e durante o período noturno.



Os meios auxiliares de locomoção são usados por 10 crianças (28\%), sendo que 2 utilizam andador com rodinhas e 8 fazem o uso apenas de cadeira de rodas (Figura 2).

Figura 2: Número de crianças que utilizam meios auxiliares de locomoção: cadeira de rodas ou andador com rodinhas.



As órteses suropodálicas sem articulação (OS) usadas em ambos os MMII eram utilizadas por 16 crianças (45\%); as órteses suropodálicas com articulação (OSA) corresponderam a 11 crianças $(31 \%) ; 7$ crianças $(20 \%)$ utilizavam órtese do tipo axilo-palmar (AP) em apenas uma mão; e 4 crianças (11\%) faziam uso de órtese áxilo-palmar nas duas mãos (APP); apenas 1 criança
(3\%) fazia uso de parapodio (PA); e 1 (3\%) de calçado sob medida (CSM). Esses dados estáo representados na Figura 3.

Figura 3: Número de crianças que utilizam cada tipo de órtese.

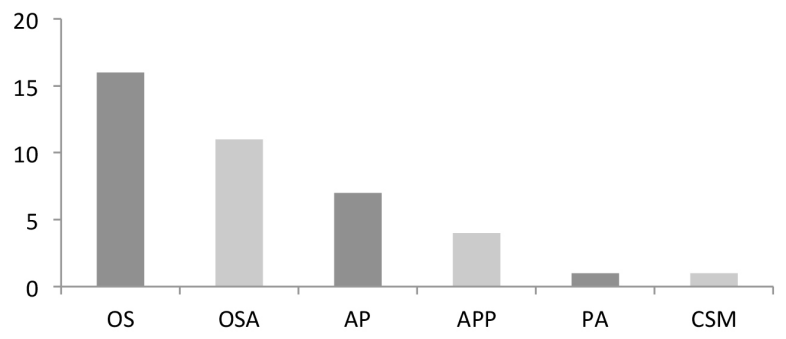

OS: órtese suropodálica sem articulação; OSA: órtese suropodálica com articulação; AP: órtese axilo-palmar em uma mão; APP: órtese axilo-palmar nas duas mãos; PA: parapodio; CSM: calçado sob medida.

\section{Discussão}

A principal alteração apresentada por crianças com PC é o comprometimento motor, com consequentes alteraçóes na biomecânica corporal, e um importante meio de auxílio para correção dessas alteraçóes são as órteses ${ }^{9}$. O suporte mecânico oferecido pela órtese suropodálica, por exemplo, visa minimizar ou corrigir o padrão equino, proporcionando melhora no padrão de marcha, com menor gasto energético e melhor qualidade de vida; essa órtese é indicada para a sustentação de um segmento do corpo ou para a inibição de movimentos involuntários, tendo como objetivos aumentar a função, prevenir deformidades e contraturas, manter as extremidades em posição funcional, auxiliar a função da musculatura fraca e facilitar o controle motor seletivo ${ }^{10}$.

No entanto, há pequena quantidade de estudos que abordam discussóes sobre o tempo de uso das órteses, o período e a relação entre o tipo e os benefícios para cada caso clínico específico. Estudos com foco nessa temática podem trazer resultados interessantes, e o conhecimento gerado está diretamente ligado à prática clínica. Em um ensaio clínico randomizado ${ }^{11}$ que visou verificar os efeitos do uso de órteses de tornozelo e pé durante o período diurno em comparaçáo ao uso do mesmo tipo de órtese durante o dia e a noite, não se obtiveram diferenças quanto ao aumento da capacidade funcional, à reduçáo de espasticidade ou ao ganho de amplitude de movimento. Por conseguinte, não há necessidade da utilização da órtese no período noturno pela criança com PC, pois isso não traria benefício significativo. No presente estudo, em que $60 \%$ das crianças fazem uso da órtese também no período noturno, o levantamento 
realizado possibilita a visualização do panorama de uso de órteses pelas crianças atendidas pelo CRF, além de intervençôes informacionais pontuais para o melhor rendimento desse meio de auxílio.

De acordo com Naslund, Sundelin e Hirschfeld, crianças com PC que fazem uso de órtese suropodálica apresentam melhora do equilíbrio durante a marcha ${ }^{12}$, afirmação que vai de encontro a Cury, Mancini, Melo, Fonseca, Sampaio, Tirado, segundo os quais o uso da órtese promove um melhor desempenho durante as tarefas motoras diárias e maior estabilidade durante a marcha de crianças com PC. Esses resultados podem ser incorporados ao presente estudo, pois tem-se um predomínio de crianças que fazem uso de órteses no período noturno, embora, de acordo com os dados apresentados, o uso fosse otimizado se feito durante o dia, para auxiliar na execução das tarefas motoras diárias.

Contraditoriamente aos resultados apresentados, uma revisão sistemática de ensaios clínicos ${ }^{13}$ concluiu que alongamentos sustentados por tempo prolongado são mais eficazes em reduzir espasticidade do que sessões diárias terapêuticas de alongamento manual; assim, cabe a interpretação de que a utilização de órteses no período noturno seria benéfica na redução da espasticidade, com consequente ganho de amplitude de movimento. Um fator que pode comprometer a transposiçáo dos resultados publicados nessa revisáo para o presente estudo é a não padronização das amostras; na revisão, foram utilizados estudos com crianças com PC e adultos pós traumatismo cranioencefálico, o que enfraquece a interpretação dos resultados.

O estudo de Kamp, Lennon, Holmes, Dallmeijer, Henley e Miller alerta sobre qual tipo de órtese é mais indicado para cada criança com base na idade, na composiçâo corporal e na mobilidade, em associação ao gasto energético durante a marcha ${ }^{14}$. No levantamento realizado no presente estudo, encontramos a utilização em larga escala de órtese suropodálica sem articulação, que não é a mais indicada para crianças com altos índices de massa corporal ${ }^{14}$. Uma das limitaçóes deste estudo foi a não captação dos dados antropométricos de cada sujeito, que poderiam incrementar a discussão sobre tipos de órteses e composição corporal. Sugere-se que futuros estudos busquem mais do que caracterizar tipos e períodos de órteses utilizadas nos centros de reabilitação e tragam dados antropométricos dos sujeitos, pois podem ser realizadas ligaçóes entre esses fatores.

Para um centro de reabilitação física o qual atenda crianças com PC é de extrema importância fazer levantamentos sobre como os responsáveis estão coordenando o uso das órteses das crianças e saber se eles estão suficientemente informados acerca de um tema tão importante no processo de reabilitação. Pensando neste problema, Gomes e Oliver realizaram uma revisão bibliográfica sobre as intervençóes realizadas em crianças com PC pela terapia ocupacional e a fisioterapia no Brasil entre 1999 e 2009, e, consequentemente, o uso de órteses é um tema amplamente abordado nesse estudo, que traz dados relevantes sobre a alienação dos responsáveis e de setores públicos de atendimento com relação ao tempo de substituição de órteses e à existência ou não de algum outro meio auxiliar capaz de aprimorar a reabilitação funcional de crianças com $\mathrm{PC}^{15}$.

Por isso, o presente estudo sugere que há a necessidade de os centros de reabilitação física registrarem em seus arquivos informaçóes sobre o tempo, o período e os tipos de órtese que seus pacientes utilizam e posteriormente publicarem esses resultados, para que o conhecimento científico sobre o tema se amplifique e para que informaçôes e guias de utilização possam ser fornecidos, de forma documentada, aos responsáveis da criança com PC.

\section{Conclusão}

O presente estudo realizou um levantamento e foi capaz de identificar que a órtese mais utilizada pelas crianças com paralisia cerebral atendidas no setor de pediatria do Centro de Reabilitação Física da Universidade Estadual do Oeste do Paraná é a suropodálica sem articulação $(45 \%)$ e que o tempo médio de permanência com a órtese é de 13 horas e 54 minutos, sendo que 60\% das crianças fazem uso da órtese no período noturno.

\section{Referências}

1. Leite JMRS, Prado GF. Paralisia cerebral: aspectos fisioterapêuticos e clínicos. Rev Neurocienc. 2004;12(1):41-5.

2. Lepage C, Noreall L, Bernard P. Association between characteristics of locomotion and accomplishment of life habits in children with cerebral palsy. Phys Ther. 1998;78(5):458-69.

3. Shepherd RB. Fisioterapia em pediatria. $3^{a}$ ed. São Paulo: Santos; 1998. p. 224-77.

4. Kooman LA, Mooney JF, Smith BP, Walker F, Leon JM. Botulinum toxin type a neuromuscular blockade in the treatment of lower extremity spasticity in cerebral palsy: a randomized, double-blind, placebo-controlled trial. J Pediatr Orthop. 2000;20:108-15.

5. Cury VCR, Mancini MC, Melo AP, Fonseca ST, Sampaio RF, Tirado MGA. Efeitos do uso de órtese na mobilidade funcional de crianças com paralisia cerebral. Rev Bras Fisiot. 2006;10(1):67-74.

6. Trombly CA. Terapia ocupacional para disfunçôes físicas. $5^{\mathrm{a}}$ ed. Sáo Paulo: Santos; 2005.

7. Fess EE. A history of splinting: to understand the present, view the past. J Hand Ther. 2002;15(2):97-132.

8. Shorter KA, Kogler GF, Loth E, Durfee WK, Hsiao-Wecksler 
ET. A portable powered ankle-foot orthosis for rehabilitation. J Rehabil Res Dev. 2011;48(4):459-72.

9. Roque AH, Kanashiro MG, Kazon S, Grecco LAC, Salgado ASI, Oliveira CS. Análise do equilíbrio estático em crianças com paralisia cerebral do tipo diparesia espástica com e sem o uso de órteses. Fisiot Mov. 2012;25(2):311-6.

10. Mattacola CG, Dwyer MK, Miller AK, Uhl TL, Mccrory JL, Malone TR. Effect of orthoses on postural stability in asymptomatic subjects with rearfoot malalignment during a 6-week acclimation period. Arch Phys Med Rehab. 2007;88(5):653-60.

11. Zhao X, Xiao N, Li H, Du S. Day vs. day-night use of ankle-foot orthoses in young children with spastic diplegia: a randomized controlled study. Am J Phys Med Rehabil. 2013;92(10):905-11.
12. Naslund A, Sundelin G, Hirschfeld H. Reach performance and postural adjustments during standing in children with severe spastic diplegia using dynamic ankle-foot orthoses. J Rehabil Med. 2007;39(9):715-23.

13. Knutson LM, Clark DE. Orthotic devices for ambulation in children with cerebral palsy and myelomeningocele. Phys Ther. 1991;71:947-60.

14. Kamp FA, Lennon N, Holmes L, Dallmeijer AJ, Henley J, Miller F. Energy cost of walking in children with spastic cerebral palsy: relationship with age, body composition and mobility capacity. Gait Posture. 2014;40(1):209-14.

15. Gomes ML, Oliver FC. The practice of occupational therapy with the child population: literature review of the period from 1999 to 2009. Rev Ter Ocup Univ. 2010;21(2):121-9. 